\title{
Configurational effects in visual information processing
}

\author{
WILLIAM P. BANKS \\ Pomona College, Claremont, California 91711 \\ and \\ WILLIAM PRINZMETAL \\ Claremont Graduate School, Claremont, California 91711
}

\begin{abstract}
These experiments show that the perceptual organization of a multielement display affects both the speed and accuracy with which a target letter in it is detected. The first two experiments show that a target is detected more poorly if it is arranged in good form (a perceptual Gestalt) with noise elements than if it is not. This effect is not confounded with target-noise proximity or display size, and it holds for stimuli terminated by the subject's response as well as for stimuli of very brief duration. Increasing the number of noise elements can actually improve performance if the added noise elements increase the degree to which the noise elements form perceptual groups separately from the target. A third experiment tries out a new method for scaling the perceptual structure of an array, and it shows that the main features of the first two experiments can be predicted from the scaled perceptual structure of the arrays they used.
\end{abstract}

In the forced choice visual detection paradigm, the subject is briefly presented an array that contains one target, chosen from a small set of target elements, and several noise elements. The subject indicates which of the target elements was presented. The main finding, called the display-size effect, is that as the number of noise elements increases, detection accuracy decreases (Estes \& Taylor, 1966; Nickerson, 1972).

Experiments on the detection paradigm have not studied phenomena of perceptual grouping such as the Gestalt "laws." Typically, stimulus configurations are constructed according to some randomization system, and effects of perceptual configuration are assumed to be, at most, a minor source of experimental noise. However, there is evidence that these configurational effects are important. Some such evidence comes from a study by Banks, Bodinger, and lllige (1974). They used arrays of elements arranged in a circle around the fixation point. Every array contained either one $F$ or one $T$, and six noise elements, the noise elements being a mixture of rectangular dot matrices and "hybrid F-Ts" (Shiffrin \& Gardner, 1972). The

This research was supported in part by a Pomona College faculty research grant to W.P.B. and by National Science Foundation Grant BMS 75-20328. The authors thank E. E. Smith and W. R. Garner for their stimulating suggestions early in the course of this research and $\mathrm{H}$. Egeth and $\mathrm{R}$. L. Gottwald for their editorial comments on an earlier draft of the manuscript. Requests for reprints should be sent to William P. Banks. Psychology Department. Pomona College. Claremont. California 91711. configuration formed by the F-Ts turned out to be the most important determiner of detection accuracy in the experiment. For example, if the F-Ts were placed together in the array and apart from the target, they interfered very little with detection accuracy, but if they formed a cluster that included the target, detection accuracy was poor. Adding F-Ts to the display actually improved performance if the additional F-Ts created a perceptual cluster that isolated the group of F-Ts from the target.

The Banks et al. study did not, however, begin as a study of configurational effects, and the conclusions are really based on post hoc analysis. The present study was therefore set up with perceptual clustering of target and noise as the independent variable. The strategy of the experiment was to begin with configurations like those seen in the column labeled "Condition 1" in Figure 1. Here the elements are arranged in a good form (Garner, 1970) and the target (an F or $T$ ) is therefore perceptually grouped with the noise elements (hybrid F-Ts). Configurations like that in the column labeled "Condition 2" were generated by adding F-Ts in such a way as to cause the noise items to be grouped separately from the target. As is seen, in Condition 2 the F-Ts form a perceptual cluster that does not include the target's position. (A study verifying that the assumed perceptual clustering does take place for the subjects is reported in Experiment III.)

The configurational hypothesis entertained here is that the mutual interfe:ence between target and noise 


\begin{tabular}{|c|c|c|c|c|c|c|c|c|c|c|c|c|}
\hline \multicolumn{13}{|c|}{ CONDITION } \\
\hline & \multicolumn{2}{|c|}{1} & \multicolumn{3}{|c|}{2} & \multicolumn{2}{|l|}{3} & \multicolumn{3}{|c|}{4} & \multicolumn{2}{|c|}{5} \\
\hline & $T$ & r & $F$ & & r & $T$ & 7 & $F$ & 7 & r & $F$ & $7 \quad 7$ \\
\hline A & & 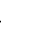 & & 7 & $=$ & $7 ?$ & & & 7 & 7 & r & 7 \\
\hline & : & 7 & 2 & 7 & $=$ & 77 & 7 & $r$ & & 7 & r & 7 \\
\hline & ${ }^{\top}$ & F & $F$ & & r & $T$ & 7 & $F$ & 7 & 7 & $T$ & 73 \\
\hline$B$ & & & & & 2 & $r$ & & & & 7 & 7 & \\
\hline & 7 & $=$ & 7 & $r$ & $=$ & $7 r$ & r & r & & 7 & 7 & r \\
\hline & $F$ & & $T$ & & & F F & & & $T$ & 7 & $F$ & 7 \\
\hline C & & & & $r$ & 7 & 77 & & & 7 & 7 & 7 & 7 \\
\hline & & 7 & & 7 & $r$ & & 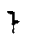 & 7 & & & & 7 \\
\hline
\end{tabular}

items is increased when they are perceptually clustered together in the same group and decreased when they are perceptually clustered in separate groups. Consequently, arrays like those in Condition 2 should give better performance than Condition 1 . The strategy of always adding noise elements to Condition 1 arrays to cause the target to be clustered separately from the noise (Condition 2) makes a conservative test of the hypothesis, since the configurational effect must work against a display-size effect. The conservatism seems necessary, however, because it is difficult to equate patterns in all respects except for the grouping structure under test, and if extraneous variables are going to be confounded with the variable of interest, it is best that they be confounded in a conservative direction.

Configurations like those of Conditions 3, 4, and 5 in Figure 1 were also presented to subjects, each as frequently as Conditions 1 and 2 , in order to reduce the possibility that the Condition 2 arrays would cue the subject as to the position of the target. Because Conditions 3, 4, and 5 place the target among the noise items, a strategy of initially searching the area of the array directly across from the mass of F-Ts would be far from optimal. Thus the subject, if he adopts a conscious search strategy, should search among the cluster of F-Ts rather than across from them, and search strategies, if they exist, should not produce spurious evidence in favor of our hypothesis.

Experiments I and II use stimulus patterns like those in Figure 1 to test for configurational effects. In Experiment I, the stimulus was left on until the subject responded, while in Experiment II, the stimulus was presented very briefly. Thus, Experiment I is a visual search experiment because the stimulus array could be scanned with eye movements by the subjects, and Experiment II is a forced choice detection experiment that limits the subject to the information available in a single fixation. Scanning strategies that require eye movements cannot, therefore, account for the results of Experiment II. The configurational hypothesis predicts that in both experiments Condition 2 will give better performance than Condition 1, which in turn will be better than Conditions 3, 4, and 5. Experiment III applies a direct measure of perceptual organization to the arrays used in Experiments I and II in order to test the assumptions about perceptual grouping on which these predictions are based.

\section{EXPERIMENT I}

\section{Method}

The subjects, on each of 600 experimental trials spread over 3 days, attempted to determine which target, always either an $F$ or a $T$, was contained in an array that had one target and a number of hybrid F-T noise elements. They were timed and asked to make their decisions as quickly as was consistent with accuracy, and the array stayed in view until they responded. The target and noise elements were arranged in patterns that varied the degree of perceptual grouping between target and noise. The principle used to vary the degree of grouring is illustrated in Figure 1, which shows how the "good form" versions (Condition 1) of patterns $A, B$, and $C$ were modified to create arrays in which target and noise elements are not grouped together (Condition 2). Conditions 3, 4, and 5 were included so that the position of the target in the array could not be predicted from the nature of the configuration. Sessions using the three patterns (A, B, and C) presented each of the conditions in equal number.

A complete stimulus set for pattern A, B, or C each contained 40 cards. This is because there are five different conditions, the target can appear in any one of four positions (or rotations of the array) in each of them, and there are two possible targets $(5 \times 4 \times 2=40)$.

The stimulus cards were printed with rubber stamps and black 
stamp-pad ink on white cards. All elements were approximately $1 \mathrm{~cm}$ wide and $1.3 \mathrm{~cm}$ high (approximately $40^{\prime} \times 52^{\prime}$ of visual angle). To reduce the possibility of lateral masking, the minimum distance between the perimeters of adjacent elements was a visual angle of $1^{\circ}$ (Gardner, 1973). Both the stimulus field and fixation fields were approximately square, subtending a visual angle of about $10^{\circ}$, both were seen at $86 \mathrm{~cm}$, and both had a luminance of about $30 \mathrm{~mL}$. The fixation cross was $16^{\prime} \times 16^{\prime}$ and was lined up with the center of all patterns.

Procedure. Each subject participated in three sessions, $1 \frac{112}{2}$ long, on consecutive days, and in each session they saw arrays based on only one of the three patterns. The order of patterns was counterbalanced over subjects. On each day, the subjects fixated the prefield cross, then after a "ready" signal depressed a footswitch. The stimulus array replaced the fixation cross $500 \mathrm{msec}$ later, and it stayed in view until the subject spoke " $T$ " or " $F$ " into a microphone that was placed about 2 in. from his mouth. The microphone was connected to a voiceoperated relay that stopped the timer and returned the fixation cross to view. The reaction time (RT) was recorded from the onset of the stimulus array. About $12 \mathrm{sec}$ after the subject's response, the experimenter said "ready" again, and the cycle was repeated.

All subjects were given one practice run through the deck of 40 stimuli at the beginning of each session. Data trials were conducted in five separate blocks of 40 each with a rest period approximately $3 \mathrm{~min}$ long between blocks. The stimulus deck was thoroughly shuffled between blocks. Stimuli that led to incorrect responses were repeated at the end of every block.

Subjects. Six students from the Claremont Colleges, four female, served as subjects in all three experiments. All subjects had normal or corrected-to-normal vision, and each was paid $\$ 9$. The subject with the fastest mean RT was paid an additional \$2, but, to discourage errors, RTs on trials that led to errors were tripled and included in the mean used to assign the award.

\section{Results and Discussion}

As is seen in Table 1, the order of RTs for patterns $\mathrm{A}, \mathrm{B}$, and $\mathrm{C}$ is as predicted by the configurational hypothesis: The RT is fastest for the configuration where noise and target group separately (Condition 2), next fastest for the good form (Condition 1), and slowest for the patterns where the target is strongly grouped with the noise
(Conditions 3, 4, and 5). Error rates were less than $1 \%$ overall and tended to fall in the harder conditions. The RTs for errors were not tabulated by condition. The main effect of condition is reliable for all three patterns, with $F(4,20)=42.53,26.87$, and 40.27 (all ps $<.001$ ) for patterns $A, B$, and C, respectively.

The most important test of the configurational hypothesis compares Conditions 1 and 2. Any theory would predict Conditions 3-5 to be worse than the others because of the display-size effect, but only the configurational hypothesis predicts a reverse display-size effect in the case of Conditions 1 and 2 . The difference between Conditions 1 and 2 is reliable overall, with an $F(1,5)$ of $23.97(p<.01)$. It goes in the predicted direction for all three patterns, but it is significant at the .01 level for only pattern A.

The results show a strong display size effect that operates in addition to the configurational effects. The three patterns, A, B, and C, contain, respectively, five, four, and three elements in their "good" version (Condition 1) and, as seen in Table 1, the RTs for this condition are ordered in accord with a display-size effect. The same ordering of RTs for the three patterns holds up for Condition 5 but not for Conditions 3 and 4 . When the data are averaged over display size without regard to configuration or patterns, RTs for displays of size 3, 4, 5, 6, and 7 are $557,573,622,643$, and $641 \mathrm{msec}$, respectively. The display-size effect was tested with a multivariate analysis of variance (MANOVA) because of the unequal Ns. This analysis showed a reliable $(p<.005)$ display size effect, with $\mathrm{F}(4,20)=5.52$.

One statistically reliable $[t(5)=6.33]$ effect in the experiment, the superiority of Condition 3 over Condition 4, seems inexplicable at this point. These conditions differ only in that the arrays in each are the four rotations of two different

Table 1

Detection Performance in Experiments I and II, and Grouping Measure From Experiment III.

\begin{tabular}{|c|c|c|c|c|c|c|}
\hline & \multicolumn{5}{|c|}{ Condition } & \multirow{2}{*}{$\begin{array}{l}\text { Independent } \\
\text { Variable }\end{array}$} \\
\hline & 1 & 2 & 3 & 4 & 5 & \\
\hline \multicolumn{7}{|l|}{ Experiment I } \\
\hline $\begin{array}{l}\text { Pattern A } \\
\text { Pattern B } \\
\text { Pattern C } \\
\text { Mean of A,B,C, }\end{array}$ & $\begin{array}{l}598 \\
573 \\
557 \\
576\end{array}$ & $\begin{array}{l}557 \\
551 \\
552 \\
553\end{array}$ & $\begin{array}{l}609 \\
638 \\
649 \\
632\end{array}$ & $\begin{array}{l}640 \\
667 \\
703 \\
670\end{array}$ & $\begin{array}{l}759 \\
735 \\
683 \\
726\end{array}$ & $\begin{array}{l}\text { Reaction time } \\
\text { of correct } \\
\text { responses } \\
\text { (msec) }\end{array}$ \\
\hline \multirow[t]{2}{*}{$\begin{array}{l}\text { Experiment II } \\
\text { (Pattern A only) }\end{array}$} & 583 & 559 & 596 & 613 & 651 & \\
\hline & 2.68 & 1.27 & 2.98 & 5.80 & 8.48 & Percent error \\
\hline \multicolumn{7}{|l|}{ Experiment III } \\
\hline $\begin{array}{l}\text { Pattern A } \\
\text { Pattern B } \\
\text { Pattern C } \\
\text { Mean of A,B,C }\end{array}$ & $\begin{array}{r}1.20 \\
1.24 \\
.34 \\
.93\end{array}$ & $\begin{array}{l}1.83 \\
1.87 \\
1.96 \\
1.89\end{array}$ & $\begin{array}{r}.95 \\
1.03 \\
.94 \\
.97\end{array}$ & $\begin{array}{l}.94 \\
.96 \\
.83 \\
.91\end{array}$ & $\begin{array}{r}.81 \\
1.04 \\
.80 \\
.88\end{array}$ & $\begin{array}{l}\text { Grouping } \\
\text { measure }\end{array}$ \\
\hline
\end{tabular}


reflections of a starting pattern. Evidence presented in Experiment III will suggest, however, that the seemingly arbitrary method for generating these two conditions created systematic differences in perceptual organization, and patterns in Condition 3 happened to isolate the target from the noise more often than those in Condition 4.

Because eye movements were possible in Experiment I, the results obtained could have been caused by the effect of the perceptual clusters on visual search strategy rather than on processes of perceptual analysis. As mentioned, the experiment was designed to promote search strategies that would, if anything, produce results opposite to those found, but it is possible that perceptual features of the array overwhelm the subject's plans in determining search patterns. Experiment II therefore replicates Experiment I with stimulus presentations too brief to allow an eye movement. Scanning strategies do not seem to affect performance in situations where brief presentations preclude eye movements (Eriksen \& Spencer, 1969; Shiffrin \& Gardner, 1972). Thus, demonstration of the same pattern of results in Experiment II as in Experiment I would show that the effects result from perceptual analysis within a single fixation rather than from strategies of visual search. Experiment II had the secondary aim of increasing error rates. Errors in Experiment I were too sporadic for meaningful analysis, and the very brief stimulus durations, as well as luminance masking by the poststimulus field, were intended to generate more errors in Experiment II.

\section{EXPERIMENT II}

\section{Method}

The experiment replicated Experiment I with three major differences: Stimulus duration was brief, only the 40 configurations based on pattern $A$ were used, and the poststimulus field was twice as bright as the stimulus to reduce visibility of the stimulus. Six subjects from the same pool as in Experiment I were used and paid $\$ 9$ for their participation, with the same instructions as before but without the monetary speed-accuracy incentive.

The experiment took about $1 \frac{1 / 2}{h}$ a day for 2 days. On the first day, the subjects were given two blocks of 40 stimuli for practice, during which the stimulus duration was reduced from about 1,000 to $50 \mathrm{msec}$. The stimulus duration was $50 \mathrm{msec}$ for all data trials and for about the last half of the practice trials. On the second day, there was a single block of practice and stimulus duration was $\mathbf{4 0} \mathrm{msec}$ for all practice and data trials. Data trials were conducted in 14 separate blocks of 40 trials each per day, giving 1,120 observations for each subject. Trials with incorrect responses were not repeated. There was a rest period of approximately $2 \mathrm{~min}$ between blocks and a rest period approximately $10 \mathrm{~min}$ long after the seventh block of trials.

Each trial began with the experimenter's "ready" signal and the subject's pressing a presentation switch. The stimulus came on $500 \mathrm{msec}$ later, was at $30 \mathrm{~mL}$ of luminance, and was followed by two fields coming on simultaneously (luminance of $60 \mathrm{~mL}$ ). The subject's verbal response stopped the clock and returned the $30-\mathrm{mL}$ fixation field (with a $1-\mathrm{mm}$-diam dot
}

fixation point) to view. Only correct RTs were recorded, and stimuli that led to incorrect responses were not repeated.

\section{Results}

As is seen in Table 1, the pattern of results, for both speed (RT) and accuracy scores, is the same for Experiment II as it was for Experiment I. For RTs, the $F(4,20)$ for the main effect of condition is 20.05 $(p<.001)$, and for errors (arcsin transformation of percent error), it is 16.97 ( $p<.001)$. The critical difference between Conditions 1 and 2 goes in the direction predicted by the configurational hypothesis for both RT and error scores, but the difference is significant only for the RTs, with $F(1,5)=10.14$, $\mathrm{p}<.025$.

The effect of the position of the target in the array turned out to have a reliable effect on both RTs and errors, with $F(3,15)$ s of 10.36 and 4.54 , respectively. For both $\mathrm{RT}$ and errors, the order of difficulty of positions is, from easiest to hardest, upper left, upper right, lower left, and lower right. This ordinal pattern of difficulty held up exactly for the RT scores for all five conditions, and with only a few reversals for the error percentages. However, the interaction between position and conditions was significant at the .01 level for both errors and RTs, with an $F(12,60)$ of 2.52 and 2.51 , respectively. The interaction arises because the position effect is stronger for the hard conditions than for the easy ones. For example, in the easiest condition, Condition 2, there is a difference of $72 \mathrm{msec}$ between the top left and bottom right positions, which are the easiest and the hardest, respectively. In Condition 5, on the other hand, the difference between these positions is $158 \mathrm{msec}$, over twice as great.

The position effect might appear to reflect scanning strategies that derive from reading habits. However, the stimulus duration was too brief to allow visual scanning, and there is evidence that purely attentional "scanning" of such briefly presented stimuli does not affect performance in a forced-choice detection experiment (e.g., Shiffrin \& Gardner, 1972). What, then, is responsible for the position effect? There seem to be at least two possible explanations. One is that subjects fixate the display somewhere in the upper left quadrant near the vertical axis, rather than at the designated central point. If they fixate in this way, and if retinal acuity falls off equally in all directions away from the fovea, then the observed positional effect will be found. However, this explanation does not cover the interaction between position and pattern difficulty. A second interpretation is in terms of perceptual grouping. The analysis of perceptual grouping presented in Experiment III indicates that the degree of grouping of target and noise varies 
with both the position of the target and the configuration, and that grouping processes may be at least partly responsible for the positional effect.

\section{EXPERIMENT III}

The present experiments have depended on an a priori system for generating configurations that have the properties that we use as independent variables. Our system seems reasonable, but it is still possible that subjects do not perceive these arrays in the way we assume. We are not alone in leaving this question unanswered; as Pomerantz and Garner (1973) point out, many investigators have altogether bypassed the problem of defining the independent variable in studies of this sort.

The question of how the visual system organizes the array should be capable of formulation in empirical terms. This experiment tries out one system for direct scaling of the perceptual organization of the arrays used here. The task for the subject is fairly simple: He is presented a copy of the array and is asked to draw boundaries around whatever clusters or groups he sees formed among the elements in it. The analysis begins by counting the number of subject-drawn boundaries that must be crossed to traverse the distance between each pair of elements in the array. The number of boundary crossings is cast into an $\mathrm{N}$ by $\mathrm{N}$ matrix, where $\mathrm{N}$ is the number of elements in the array, and the number in the cell ij is the number of boundary lines between elements $i$ and $j$. The perceptual structure could be recovered from a matrix of this type by means of a hierarchical clustering analysis (Johnson, 1967), but, for the present study, only a measure of the degree of grouping between target and noise is needed. The measure used here is the mean number of boundaries between the target and the noise elements. The more such boundaries there are, the more isolated is the target from the noise and the better performance should be.

\footnotetext{
Method

The subjects, who performed together in one group of 21, were each given copies of the stimuli used in Experiment $I$ and were asked to draw boundaries around items that seemed to group together. The instructions illustrated the task with a group of three small circles arranged in an oblique triangular configuration. The instructions showed how the three circles could be seen as a single triangle, a horizontal group of two circles in a line with a single circle lying above, and so on, and the boundaries that should correspond to each structure were illustrated in each case. It was explained that there were no right or wrong answers and that each person should simply draw lines to indicate the perceptual groupings of items as he or she saw them. No restrictions as to the number of lines or the crossing or nesting of boundaries were made.

Two sets of stimulus material were used, each with 60 stimulus patterns. The patterns were enclosed in a rectangle approximately $5 \times 5.5 \mathrm{~cm}$ and were printed 12 to an $81 / 2 \times 11$ in.
}

page. The stimulus material was dittoed from 50\% Xerographic reductions of the stimulus cards used in Experiment 1 . Each set of stimulus material contained one example from each configuration and target position with target ( $T$ or $F$ ) determined by chance, but if a given stimulus pattern in one set contained a $T$, the other contained an $F$.

Since there were 21 in the group of subjects (students in a psychology class at Pomona College, none of whom had served in Experiment I or Experiment II), 11 got one set and 10 the other. The protocol of one subject was dropped from the set given to the 11 subjects to equalize the amount of data in each set, but as it turned out, this protocol was not chosen at random. Rather, it was impossible to score and would have had to be dropped from analysis anyway. None of the other protocols presented any such unexpected problems in analysis.

\section{Results and Discussion}

The grouping measure was calculated for each subject's array by adding up the number of lines between the target and each noise element and then dividing this sum by the total number of noise elements in the array. The measure is therefore the mean number of boundaries that separate the target from the average noise element, and it should be, at least roughly, a measure of the degree to which the target and noise items are isolated from each other perceptually. The higher the grouping index, the more isolated is the target from the noise, and the better performance should be.

An analysis of variance identical in form to that used in Experiment I was conducted, with grouping score rather than $\mathrm{RT}$ as the dependent variable. The three pattern sets turned out to differ reliably overall beyond the .01 level, with an $F(2,38)=$ 9.27, and with mean grouping scores of 1.15 , 1.23 , and 0.97 for sets $A, B$, and $C$, respectively. The five conditions also differed significantly, with $F(4,76)$ of 54.5 , and the differences among the conditions in grouping generally paralleled the $R T$ and error data ( $\mathrm{r}=-.65$ overall). In particular, the difference in grouping between Conditions 1 and 2 was highly significant, with an $F(1,19)=77.3$. and mean grouping scores of .93 and 1.89 , respectively. Thus, the boundary-drawing results show that the target has about twice as many boundaries between it and the average noise item in Condition 2 as in Condition 1. These results (fortunately) vindicate our initial predictions about grouping and our intuitive sense of the grouping structure of the arrays. At the very least, we can assert now that there is an empirical basis for our main prediction about the effect of perceptual structure on performance. The relationship between RT and the grouping measure (seen in Table 1) is quite consistent across patterns, with the exception of pattern $\mathrm{C}$, Condition 1. The correlation between $\mathrm{RT}$ and grouping is -.52 . The correlation would undoubtedly be better if some nonlinear transformation of the grouping measure were used, since the 
relationship between the two variables is roughly an exponential one. Error rates have a - .76 correlation with the grouping measure.

The peculiar and seemingly inexplicable superiority of Condition 3 over Condition 4 in both RTs and errors can be explained in terms of perceptual grouping, since Condition 3 shows a greater degree of separation of target and noise than does Condition 4. This difference holds up for all three patterns but falls short of statistical significance, with an $F(1,19)=3.1, .05<p<.10$.

A separate analysis of variance was performed on the grouping measures for pattern $\mathrm{A}$ alone in order to provide a comparison with Experiment II. Again, the five conditions differed reliably, with $F(4,76)=$ 24.1. The grouping scores for the critical conditions, 1 and 2 , were 1.20 and 1.83 , respectively, with $\mathrm{t}(18)=3.78, \mathrm{p}<.01$, and they therefore had the predicted inverse relationship to the RTs and errors.

The position of the target in the array had little consistent overall effect on its degree of grouping with the noise $(F=.54)$. However, the interaction between position and condition approaches significance, with $F(12,228)=1.61$, and the grouping scores broken down by position and condition show a pattern that predicts the pattern of both RTs and errors over condition and position in Experiment II: $\mathrm{r}=-.43$ for $\mathrm{RTs}$ and -.51 for errors. It is interesting, incidentally, that the regression between grouping and error rate predicts an error rate of $53 \%$ when the grouping score is zero. That is, the regression predicts that performance falls approximately to chance when the target and noise are perfectly grouped together. The other regressions between errors and grouping make a similar prediction, but this one is the most reliable, since it uses the error data from Experiment II and correlates over all of the experimental variab'es.

\section{GENERAL DISCUSSION}

The present studies show several different ways in which organization affects visual processing. In Experiment I, eye movements were possible, and the effects could be attributed to strategies of visual search, but in Experiment II, the short stimulus presentations precluded overt visual scanning, and the effects there must be the result of processes of perceptual analysis that operate on the information available in a single fixation. Since the direction and size of the configurational effect is about the same in both experiments, it seems likely, in fact, that strategies of visual scanning played only a small role in creating the effects of Experiment $I$.

The results of the grouping study provide, at the least, the logical foundation for concluding that organization affects processing, since the grouping measures give the necessary independent variable from which to predict RT and error scores. Without such an independent variable, predictions must be based on intuitive notions that do not follow from explicit operational definitions and are very difficult to incorporate into a theoretical account of the phenomena. The grouping measure, as it turned out, did verify the initial intuitive assumptions about the processing effects of Conditions 1 and 2 , and it also accounted for two unexpected findings: (1) the RT and error differences between Conditions 3 and 4 , and (2) the Position by Condition interaction. Thus, the grouping measure showed its worth in more ways than one, because it showed processing consequences of aspects of perceptual structure that are not obvious on an intuitive level.

A single generalization characterizes all of the effects of organization on processing reported here. This is that the more the target is perceptually grouped with the noise, the more it is interfered with by the noise. This generalization underlies the initial prediction of the effects of Condition 1 vs. Condition 2, and it also underlies the predictions based on the grouping measure; in both cases, it is verified by the results. Another way of putting this generalization is to say that if the target and noise come in on the same "channel" they will interfere with each other more than if they are in separate channels. The analogy with auditory selective attention is apparent. If one of two similar, simultaneously presented auditory messages is to be shadowed, performance will improve as the spatial separation between the messages is increased, or as any other difference between them is introduced that allows organization of them into separate channels of information before analysis for meaning. Likewise, performance in this visual processing task will vary with the degree to which the target and noise are organized into separate sources of information. The stimulus characteristics that determine channel formation in listening are fairly well known; they include such factors as differences in pitch and spatial location of the voices. In visual processing, the Gestalt principles of organization summarize many of the stimulus factors in visual "channel formation." It should be emphasized that in neither the visual nor the auditory case is it understood why some stimulus characteristics determine channel formation and others do not, even though we do have extensive lists of these characteristics for both modalities.

A theoretical model of processing that covers the present organizational effects seems to have two tasks before it. First, it should explain how the configuration, similarity structure, and other stim- 
ulus factors determine the perceptual organization of the array. Second, it should explain how perceptual organization, once it is created, affects processing. There are a number of different ways of accounting for these two aspects of processing, but it seems best to locate them in separate information processing stages. The first of these stages would be a perceptual "parser" and the second an analyzer. The parser would organize information in parallel from all over the field into groups, and the groups it formed would be determined by such factors as the Gestalt principles. The analyzer would operate on the information at the level of the groups formed by the parser. It is premature to specify the mechanism by which the analyzer works; it might be either serial or parallel, and there are several models that might fit the data under the present assumptions. The important assumption for the present purposes is only that the analyzer operates on groups that are output by the parser. Thus, performance of the analyzer would be poor if the target were grouped with confusable noise elements or if groups were parsed at the wrong level of analysis for the task. If the stimulus is available for a sufficient duration, the analyzer may signal the parser to reorganize the input if it does not find the target. Kahneman (1973) has shown the plausibility of such a recursive loop, by which "unit formation" (parsing) may be directed by more central processes, and it seems reasonable to leave open the possibility of reparsing here.

A model along this line can explain the present results as well as those of Banks et al. (1974) and several other findings in the literature. The similarities among the elements would operate in different ways at the two levels in the theory. At the level of the parser, element similarity would operate, by the principle of similarity grouping, to determine which elements would be grouped with which other elements into channels. Thus, noise elements that are similar to each other would group separately from the target and therefore not interfere with it. The improvement in target detection with noise redundancy (McIntyre, Fox, \& Neale, 1970; cf. also Banks et al., 1974) would thus arise from processes of early perceptual organization that cause the target and noise to group separately, and that consequently protect the target from inter- ference. By the same argument, if noise elements are similar to the target, they will group with it and interfere with it. A combination of these two processes could lead to the phenomenal "jumping out" of the target in visual search when the target and distractors are dissimilar (Neisser, 1967, especially Chap. 3). At the second level in the model, after perceptual channels are formed by the parser, target-noise similarity would influence performance by affecting decision processes, as in Gardner's (1973) or Kinchla's (1974) models. Presumably, the interference with target detection at this level would come from noise elements clustered in the same perceptual group with it.

\section{REFERENCES}

Banks, W. P., Bodinger, D., \& Illige, M. Visual detection accuracy and target-noise proximity. Bulletin of the Psychonomic Society, 1974, 2, 411-414.

Eriksen, C. W., \& SPEncer, T. Rate of information processing in visual perception: Some results and methodological considerations. Journal of Experimental Psychology Monographs, 1969, 79(No. 2, Part 2).

Estes, W. K., \& TAYloR, H. A. Visual detection in relation to display size and redundancy of critical elements. Perception \& Psychophysics. 1966. 1, 9-16.

GARDNER, G. T. Evidence for independent parallel channels in tachistoscopic perception. Cognitive Psychology, 1973, 4. 130-155.

GARNER, W. R. Good patterns have few alternatives. American Scientist, 1970, 58, 34.42.

Johnson, S. C. Hierarchical clustering schemes. Psychometrika, $1967,32,241-254$.

Kahneman, D. Attention and effort. Englewood Cliffs, N.J: Prentice-Hall, 1973.

Kinchla, R. A. Detecting target elements in multi-element arrays: A confusability model. Perception \& Psychophysics, 1974, 15, 149-158.

McIntyre, C., Fox, R., \& Neale, J. Effects of noise similarity and redundancy on the information processed from brief visual displays. Perception \& Psychophysics, 1970, 7, 328-332.

NeIsser, U. Cognitive psychology. New York: Appleton-CenturyCrofts, 1967.

Nickerson, R. S. Binary classification reaction time: A review of some studies of human information-processing capabilities. Psychonomic Monograph Supplement. 1972, 4(17), 275-318.

Pomerantz, J. R., \& Garner, W. R. Stimulus configuration in selective attention tasks. Perception \& Psychophysics, 1973, 14, 565.569.

Shiffrin, R. M., \& Gardner, G. T. Visual processing capacity and attentional control. Journal of Experimental Psychology. 1972, 93, 72-82.

(Received for publication December 1, 1975; revision received January 26,1976 .) 